\title{
Tuning rectangular microstrip antenna through scan and zoom algorithm
}

\author{
Qinglian Zhang ${ }^{1,2}$, Xin Zhang ${ }^{1,2^{*}}$ (D) and Yupeng Li ${ }^{1,2}$
}

\begin{abstract}
Microstrip antennas have been widely studied and can be applied to a large number of wide frequency domain in wireless communication. Microstrip antennas are featured with light weight, small volume, and low cost advantages. Planar or spatial structures could substantially affect the frequency reliability and sidelobe level of microstrip antennas. Designing high performance microstrip antennas requires the tuning of the length and width of microstrip patch, the equivalent permittivity, the feed position of the coaxial line, and so on. Although electromagnetic theory could provide a solution for antenna design, such solution is probably not optimal solution due to complex environment and the affection of multiple factors. This paper attempts to tuning the design of microstrip antennas by using scan and zoom algorithm. It does not require derivative information of antenna model. This algorithm consists of search space scan and search domain zoom stages. The effectiveness of the scan and zoom algorithm is firstly tested on mathematical functions. The algorithm is then verified on rectangular microstrip antenna design. Compared with theoretical solution, the novel algorithm attains better design solution. The algorithm can also be easily extended to tackle other antenna design problems.
\end{abstract}

Keywords: Microstrip antenna, Scan and zoom, Antenna design, Numerical optimization

\section{Introduction}

Radar sensor networks usually consist of a transmitting node and several receiving nodes. It is very useful in the context of passive localization and tracking. As a wireless network system, antenna arrays and radar elements are important to increase the diversity gain and reduce the probability of miss detection and false alarm [1-3]. How to design the antenna shape and deploy array elements is a science and art. Different designing methods and deploying manners are proposed to accurately detect targets [4-6].

The concept of microstrip antenna is first proposed in 1950s. With the development of a good model and application of copper and gold deposited dielectric substrate lithography technology, Munson and Howell made the earliest microstrip antenna in early 1970s. The advantages of microstrip antenna are light weight, small volume, low cost, convenient to connect with integrated circuit

\footnotetext{
*Correspondence: ecemark@mail.tjnu.edu.cn

'Tianjin Key Laboratory of Wireless Mobile Communications and Power Transmission, Tianjin Normal University, Tianjin, China

${ }^{2}$ College of Electronic and Communication Engineering, Tianjin Normal University, Tianjin, China
}

and carrier, and so on. It has been deeply researched and applied to a large number of $100 \mathrm{MHz}-100 \mathrm{GHz}$ wide frequency domain on radar and radio equipments. In the field of microwave antenna, microstrip antenna has formed its own independent research direction.

To fulfill the need of wireless communication in radar sensor networks, ad hoc networks, or other wireless network systems $[7,8]$, it is necessary to design high gain, wideband, and reliable microstrip antennas. Generally, the design problem is resolved from two directions. One direction is to construct new antennas based on electromagnetic theory $[9,10]$. The other is to simulate antenna model on computer and use optimization algorithms to design high performance antennas. Optimization algorithms are divided into deterministic algorithms and stochastic algorithms [11]. Deterministic algorithms such as gradient descent and Newton method converge very fast, though they require derivatives of objective functions [12-14]. Yu et al. studied a decomposition method for bound constrained optimization problems [15]. Stochastic algorithms such as evolutionary algorithms, particle swarm optimization $[16,17]$, and artificial bee colony converge slower than deterministic ones [18-20] though they

\section{Springer Open}

(c) The Author(s). 2018 Open Access This article is distributed under the terms of the Creative Commons Attribution 4.0 International License (http://creativecommons.org/licenses/by/4.0/), which permits unrestricted use, distribution, and reproduction in any medium, provided you give appropriate credit to the original author(s) and the source, provide a link to the Creative Commons license, and indicate if changes were made. 
take objective functions as black-box, which makes these algorithms applicable to nearly all kinds of antenna design problems. Parallel execution of optimization algorithms could greatly save computational time [21, 22]. Hence, stochastic algorithms are becoming more and more popular in antenna designings.

Traditional deterministic methods or manual adjustment methods could not straightforwardly solve such antenna design problems. Thus, this study first constructs an optimization model for microstrip antenna. Second, we use scan and zoom algorithm to perform optimization. It is a heuristic algorithm and does not require derivatives of objective functions. Hence, it is applicable to simulationbased design problems. Scan and zoom algorithm consists of two main stages: (1) search space scan and (2) search domain zoom. Search space scan stage explores the whole search space and aims to find the domain of global optimum. Search domain zoom stage determines which domain worth more scanning and aims to refine current best solution. The advantages of scan and zoom algorithm are simple to implement and easy to use and does not introduce random factors. The proposed design framework is verified on both mathematical functions and rectangular microstrip antenna design problems.

In Section 2, the design of microstrip antenna and related works are presented. Section 3 gives the scan and zoom algorithm and the proposed design framework. Section 4 presents numerical results and discussions. The paper is concluded in Section 5.

\section{Microstrip antenna design}

Since 1970s, microstrip antenna has been widely studied; many design methods have been created and proposed. As shown in Fig. 1, a microstrip antenna is typically made up of a radiation patch attached to a dielectric substrate with a metal floor. Patch conductors are usually copper, aluminum, gold, etc. The shape of a patch can be arbitrary, but ordinary shape is the first choice for analysis simplification and performance evaluation.

Consider rectangular microstrip antenna as in Fig. 1, suppose the origin locates in the center of rectangular patch and establish coordinate system. Denote that $l$ and

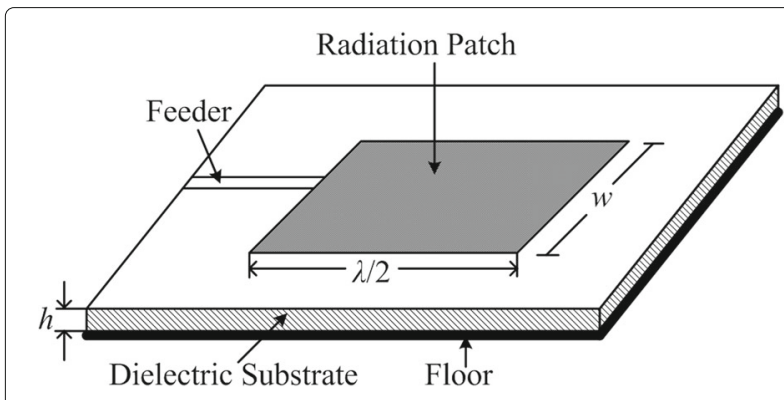

Fig. 1 Structure of microstrip antenna $w$ are the length and width of radiation patch. Denote that $h$ is the thickness of substrate. Clearly, radiation patch, substrate, and ground pad can be equivalent to a low impedance microstrip transmission line with length $l$. It is an open circuit due to breaking off on both sides of transmission line. Based on equivalence principle, the design procedure is to determine $l$ and $w$ given $\epsilon_{r}, f_{0}$, and $h$, where $f_{0}$ represents the operating frequency of antenna and $\epsilon_{r}$ is the relative dielectric constant of the medium.

The width $w$ of the rectangular microstrip patch can be calculated by the formula:

$$
w=\frac{c}{2 f_{0}} \sqrt{\frac{2}{1+\epsilon_{r}}},
$$

where $c$ represents the free-space velocity of light.

According to (1), given $\epsilon_{r}=2.25, c=3.0 \times 10^{8} \mathrm{~m} / \mathrm{s}, f_{0}=$ $3 \mathrm{GHz}, w$ is approximately equal to $39.2 \mathrm{~mm}$. Denote $w^{*}=$ $39.2 \mathrm{~mm}$. In general, when the width is chosen smaller than $w^{*}$, the radiation efficiency of the patch becomes lower; on the other hand, when the width is chosen greater than $w^{*}$, the radiation efficiency becomes higher, though it causes high changes of the distortion of the field.

The length $l$ of the rectangular microstrip patch can be calculated by the formula:

$$
l=\frac{c}{2 f_{0} \sqrt{\epsilon_{\mathrm{eff}}}}-2 \triangle l
$$

where $\epsilon_{\text {eff }}$ represents equivalent permittivity and $\Delta l$ is the length of equivalent radiation gap. The equivalent permittivity $\epsilon_{\text {eff }}$ can be calculated in accordance with the formula:

$$
\epsilon_{\mathrm{eff}}=\frac{\epsilon_{r}}{2}+\frac{\epsilon_{r}-1}{2}\left(1+\frac{12 h}{w}\right)^{-\frac{1}{2}} .
$$

In (3), given $\epsilon_{r}=2.25, w=w^{*}=39.2 \mathrm{~mm}$, and $h=5 \mathrm{~mm}, \epsilon_{\text {eff }}$ is about equal to 2.018 . For the equivalent radiation slot length $\Delta l$, it can be calculated according to the formula:

$$
\triangle l=0.412 h \frac{\left(\epsilon_{\text {eff }+0.3}\right)(w / h+0.264)}{\left(\epsilon_{\text {eff }}-0.258\right)(w / h+0.8)} .
$$

In (4), given $\epsilon_{\mathrm{eff}}=2.018, w=w^{*}=39.2 \mathrm{~mm}$, and $h=5 \mathrm{~mm}, \Delta l$ is about equal to $2.55 \mathrm{~mm}$. Hence, in (2), given the above $c, f_{0}, \epsilon_{\mathrm{eff}}$, and $\Delta l, l$ approximates to $30 \mathrm{~mm}$.

Based on traditional theoretical analysis, $w^{*}=39.2$ and $l^{*}=30$ are optimal solution for such a rectangular microstrip antenna design. Next, the design is modeled as a constrained optimization problem as follows:

$$
\begin{array}{ll}
\min _{w, l, h} S(w, l, h) \\
\text { s.t. } & f_{R}=3 G H z \\
& 29.2 \mathrm{~mm} \leq w \leq 49.2 \mathrm{~mm} \\
& 20 \mathrm{~mm} \leq l \leq 40 \mathrm{~mm} \\
& 1 \mathrm{~mm} \leq h \leq 11 \mathrm{~mm}
\end{array}
$$


where $w, l$, and $h$ are parameters to be tuned; $f_{R}$ is the resonant frequency of the antenna; and $S(w, l, h)$ is the scatting parameter of the antenna. By minimizing $S$ and assuring $f_{R}=f_{0}=3 \mathrm{GHz}$, the design of rectangular microstrip antenna can be optimized.

Sobhani et al. proposed a tracking algorithm based on low-complexity particle filtering specifically tailored to radar sensor networks with one transmitter and several receivers [23]. Through studying the coverage problem of a bistatic radar sensor network, Gong et al. reported valuable insights into the placement of bistatic radars for barrier coverage [24]. Liang and Cheng proposed a knowledge-based persistent sensor network for estimating parameters in target radar cross section [25]. Han et al. analyzed relaying in multi-hop networks [26]. Alam et al. studied multiband microstrip patch antenna based on splitting method [27].

Chen et al. studied a low-profile and wide-beamwidth dual-polarized distributed microstrip antenna [28]. The proposed antenna operated at 2 and $2.2 \mathrm{GHz}$. Frequency bands and beamwidth were optimized by tuning positions of micro patches. Liu et al. studied a low-profile differential-fed dual-polarized microstrip antenna [29]. The proposed antenna operated at 1.71 to $1.85 \mathrm{GHz}$. It used a differentially feeding scheme to suppress evenorder modes. Bandwidth enhancement was achieved by symmetrically arranging an array of shorting pins. The study in this paper differs from these researches in two aspects. First, antenna operating frequency is different. This paper focuses on $3 \mathrm{GHz}$ microstrip antenna, though the method can also be expanded to other frequency. Second, the above two researches tune antenna mostly based on empirical experience, which could hardly be extended to handle other microstrip antenna types. This paper attempts to use scan and zoom algorithm to do optimization, which saves manual tuning of antenna parameters.

\section{The used algorithm}

The scan and zoom algorithm is given in this section as well as the proposed optimization framework.

\subsection{Scan and zoom algorithm}

Scan and zoom optimization algorithm (SZOA) was proposed by Venkataraman [30]. As shown in Fig. 2, the algorithm consists of two stages: (1) search space scan and (2) search domain zoom. At the scanning stage, sampling is done over whole search space. Alike to traditional nonlinear programming methods [31-33], it is a deterministic operation without any random factors. The SZOA method is derivative free compared with Newton-type methods [34, 35]. Zoom stage is subdivided into narrowing search scope and enlarging the scope of search, which is based on the state of current optimal solution.

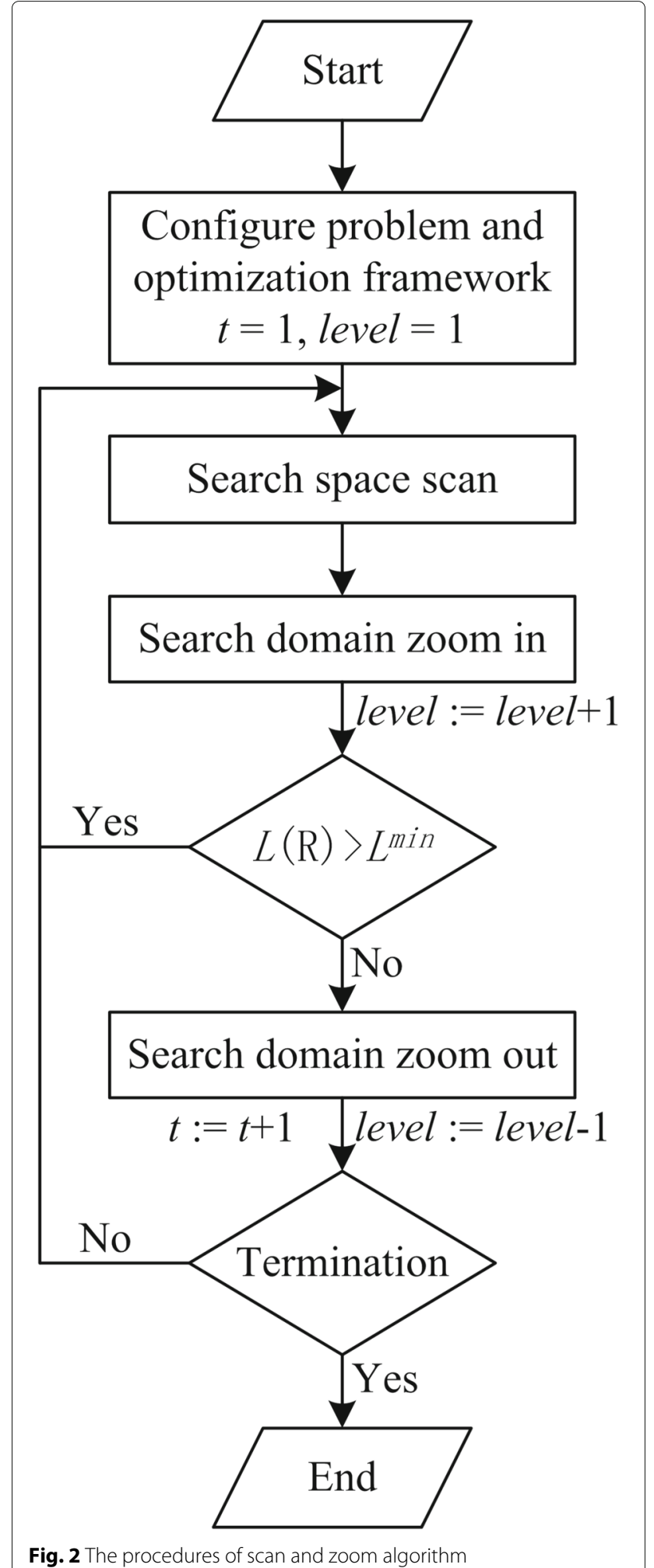

The problem to be solved is considered as a black-box, which reduces the calculation operation of random number and improves the efficiency of execution compared with evolutionary algorithms. 
The procedures of SZOA, as shown in Fig. 2, includes the following steps:

Step 1: Initialize the SZOA method and design problem including the parameters and methods that need to be specified. For example, the search space $\Omega$, the number of variables $D=3$, the number of sampling points $N$, the iteration variable $t=1$, the scanning layer number level $=1$, and the objective function $S$.

Step 2: Scan stage. Ns sample points are taken with equal intervals in each dimension of the search space $R$, and at the initial time $R=\Omega, R$ is divided into $(N s-1) D$ regions to form NsD sampling points. The sample points are evaluated based on model (5). Sort these points according to the minimization criterion, and mark each region formed by segmentation according to order.

Step 3: Zoom in stage. The point with minimal function value is selected as the best choice at present. The region containing the point in the boundary as the reduced feasible space $R$, and mark the area corresponding to the search area has been searched.

Step 4: If the length of the feasible space is greater than $L_{\text {min }}$, then the search subdivides one level := level +1 and turn to step 2; otherwise, the area corresponding to the marked feasible space is searched and turn to step 5 .

Step 5: Zoom out stage. If the current level is still not the search area, then select all the sample points in the region without minimum point as the current best choice in the point boundary region as the feasible space $R$, and mark the space corresponding to the area for search, go to step 2; otherwise, level returns the previous layer of level $:=$ level -1 , in case all regions have be searched, go to step 6 .

Step 6: If all regions have been searched or met the pre-specified termination conditions, the method is terminated and output the best solution; otherwise, turn to step 2.

\subsection{The proposed optimization framework}

The design problem shown in (5) is a simulation-based model, which may be impossible using traditional optimization methods [36, 37]. Thus, an optimization framework is proposed by solving design problem based on SZOA. The framework is shown in Fig. 3.

The optimization framework is comprised by two parts. One is SZOA method and the other is microstrip antenna system. The former decides the parameter values for antenna size and shape and refines the fitness of parameter values in each iteration. The latter builds antenna system based on given parameter values, simulates the working of antenna system, and evaluates the efficiency of the resulting antenna.

As to algorithmic parameters of SZOA method, large $N s$ causes high explorative ability of the algorithm, whereas small $N s$ reduces such ability of searching whole space. Moreover, SZOA with larger level has better exploitative ability than smaller value, whereas computational time also increases greatly as the increase of level.

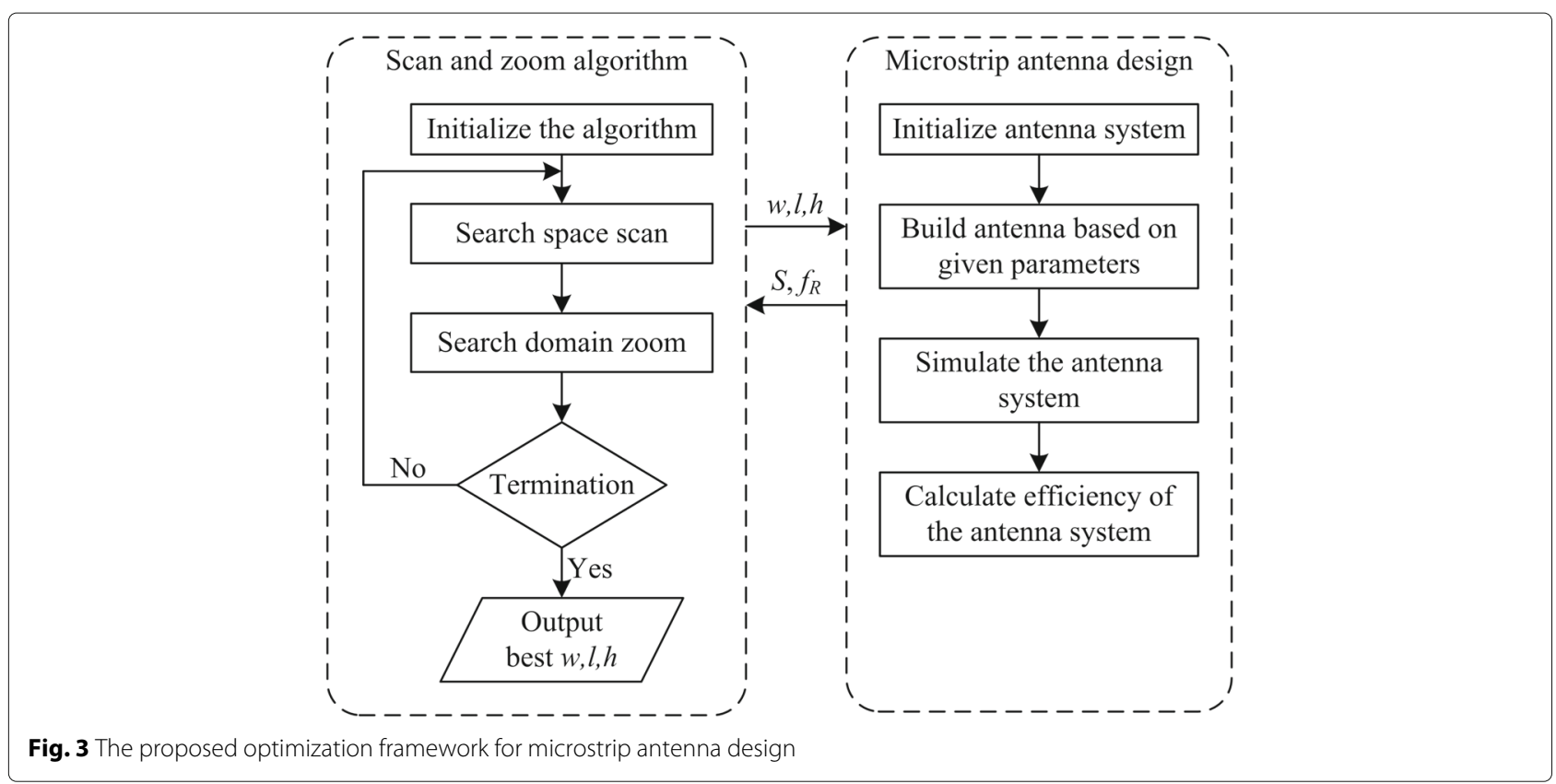




\section{Results and discussion}

In the numerical simulation, the SZOA algorithm is first tested on mathematical functions and compared with three other methods. They are pattern search (PS) [38], continuous non-revisiting genetic algorithm (cNrGA) [39], and hybrid chaotic quantum particle swarm optimization (HCQPSO) [40]. For SZOA, Ns = $\left\lfloor 10^{\lg (M F E N / D) / D}\right\rfloor$ and $l^{\min }=10^{-6}$, where MFEN represents the maximum function evaluation numbers. For PS, cNrGA, and HCQPSO, they are initialized by the default settings as in their papers. A set of six mathematical functions are taken as the first study. Functions as well as their properties are listed in Table 1. This table shows two unimodal functions and four multimodal functions used in simulation. $D$ is set to 10 for these test functions. The SZOA algorithm is then tested on rectangular microstrip antenna design as shown in Section 4.2.

\subsection{Results on mathematical functions}

Table 2 shows the statistics of optimal objective function values attained by the four test algorithms. It can be seen from Table 2 that all algorithms find global optimum of $f_{1}$. For the other functions, the SZOA algorithm attains smaller values than the PS, cNrGA, and HCQPSO algorithms. Compared PS with SZOA, it can be seen that for all functions, standard deviation (std) values of both algorithms are 0 . This is because both algorithms do not contain random factors; hence, the same results are reached in each run. cNrGA and HCEPSO are two state of that art algorithms, though they are outperformed by the SZOA algorithm. For the multimodal functions, cNrGA and HCQPSO show large variance, on the other hand, SZOA not only finds smaller values, but also is very stable. Thus, it is able to conclude that the proposed optimization framework could be effective for the design of microstrip antenna design.

\subsection{Results on microstrip antenna design}

As in Section 2, $w^{*}=39.2 \mathrm{~mm}, l^{*}=30 \mathrm{~mm}$, and $h=5 \mathrm{~mm}$ are optimal setting based on electromagnetic theory. The antenna design is optimized by the proposed

Table 1 Mathematical functions: two unimodal functions and four multimodal functions

\begin{tabular}{lll}
\hline Number & Function & Property \\
\hline$f_{1}$ & Spherical function & Unimodal, separable, scalable \\
$f_{2}$ & Different power function & Unimodal, separable, scalable \\
$f_{3}$ & Rastrigin function & Multimodal, separable, scalable \\
$f_{4}$ & Rosenbrock function & Multimodal, nonseparable, scalable \\
$f_{5}$ & Ackley function & Multimodal, nonseparable, scalable \\
$f_{6}$ & Rotated Schwefel function & Multimodal, nonseparable, scalable \\
\hline
\end{tabular}

optimization framework. The same parameter setting is used as in previous subsection.

First, let fix $w=w^{*}$ and $h=5 \mathrm{~mm}$ and set $l$ as a parameter to be optimized by the proposed framework. Figure 4 shows the results compared theoretical solution and optimized solution. It can be seen from the figure that the SZOA algorithm finds better solution than the one computed based on theory. The parameter $l$ is $30.25 \mathrm{~mm}$ which is slightly greater than $l^{*}$. In terms of objective function $S(w, l, h)$, theoretical solution is improved by $17.71 \%$.

Second, set $w, l$, and $h$ as three parameters to be optimized by the proposed framework. Figure 5 shows the results compared theoretical solution and optimized solution. It can be seen from the figure that the SZOA algorithm finds better solution than the one computed based on theory. The optimal parameters for $w, l$, and $h$ are respectively $40.2,30.125$, and $5.375 \mathrm{~mm}$. All values are greater than theoretical values. In terms of $S(w, l, h)$, theoretical solution is improved by $61.56 \%$.

Therefore, the proposed framework is effective to solve microstrip antenna design. Moreover, theoretical solution is greatly improved by SZOA, which is verified both on one parameter and three parameters optimization.

\section{Conclusions}

Recently, radar sensor networks have been more and more widely used in various fields [41, 42]. To achieve good network stability and throughput of data transmission, antenna design is worthy of studying as it is a difficult and time-consuming task. Microstrip antenna design is considered in this paper. Different from existing approaches, this paper uses the SZOA algorithm to optimize the design problem. The contribution of this paper is twofolds. First, antenna design is formulated as a minimization problem (5). It is a simulation-based optimization problem; hence, stochastic algorithms should be used. Second, an optimization framework is proposed based on the SZOA algorithm. This algorithm does not use derivative information and does not involve random factors.

The usefulness of the SZOA algorithm is shown on six mathematical functions including two unimodal functions and four multimodal functions. It turns out that SZOA performs better than PS, cNrGA, and HCQPSO methods. There is no need to execute SZOA repeatedly as it returns the same result given the same setting. Moreover, the proposed framework is applied to tackle rectangular microstrip antenna design. Compared with theoretical optimal setting, the proposed framework can find better solution.

An overhead of the SZOA algorithm is that it requires sampling of each parameter, while the number of samples grows quickly with the increase of parameters. It is suggested to solve problems with less than 10 parameters. 
Table 2 Optimal function values found by PS, cNrGA, HCQPSO, and SZOA over 25 independent runs

\begin{tabular}{|c|c|c|c|c|c|}
\hline$f(\cdot)$ & Algorithm & $\min$ & med & $\max$ & std \\
\hline \multirow[t]{4}{*}{$f_{1}$} & PS & 0.0000 & 0.0000 & 0.0000 & 0.0000 \\
\hline & $\mathrm{cNrGA}$ & 0.0000 & 0.0000 & 0.0000 & 0.0000 \\
\hline & HCQPSO & 0.0000 & 0.0000 & 0.0000 & 0.0000 \\
\hline & SZOA & 0.0000 & 0.0000 & 0.0000 & 0.0000 \\
\hline \multirow[t]{4}{*}{$f_{2}$} & PS & $8.600 E-3$ & $8.600 E-3$ & $8.600 E-3$ & 0.0000 \\
\hline & $\mathrm{cNrGA}$ & $1.369 \mathrm{E}-4$ & $2.870 \mathrm{E}-3$ & $2.560 \mathrm{E}-3$ & 0.0077 \\
\hline & HCQPSO & $7.250 \mathrm{E}-5$ & $1.740 \mathrm{E}-4$ & $2.850 \mathrm{E}-4$ & 0.0001 \\
\hline & SZOA & $3.210 \mathrm{E}-5$ & $3.210 \mathrm{E}-5$ & $3.210 \mathrm{E}-5$ & 0.0000 \\
\hline \multirow[t]{4}{*}{$f_{3}$} & PS & 2.308E2 & 2.308E2 & 2.308E2 & 0.0000 \\
\hline & $\mathrm{cNrGA}$ & 9.171EO & 1.039E1 & 1.989E1 & 0.6293 \\
\hline & HCQPSO & 9.660EO & 1.863E1 & 3.404E2 & 7.7001 \\
\hline & SZOA & 8.955E0 & 8.955E0 & 8.955E0 & 0.0000 \\
\hline \multirow[t]{4}{*}{$f_{4}$} & PS & 4.560EO & 4.560EO & 4.560Е0 & 0.0000 \\
\hline & $\mathrm{cNrGA}$ & $1.266 \mathrm{E}-1$ & 3.280E1 & 7.460E1 & 32.3284 \\
\hline & HCQPSO & $3.440 \mathrm{E}-2$ & 6.260 E0 & 1.008E1 & 4.7548 \\
\hline & SZOA & $5.490 \mathrm{E}-4$ & $5.490 \mathrm{E}-4$ & $5.490 \mathrm{E}-4$ & 0.0000 \\
\hline \multirow[t]{4}{*}{$f_{5}$} & PS & 2.059E1 & 2.059E1 & 2.059E1 & 0.0000 \\
\hline & $\mathrm{cNrGA}$ & 2.027E1 & 2.035E1 & 2.045E1 & 0.0774 \\
\hline & HCQPSO & 2.024E1 & 2.039E1 & 2.054E1 & 0.0790 \\
\hline & SZOA & 2.023E1 & 2.023E1 & 2.023E1 & 0.0000 \\
\hline \multirow[t]{4}{*}{$f_{6}$} & PS & 1.840 E3 & 1.840E3 & 1.840 E3 & 0.0000 \\
\hline & $\mathrm{cNrGA}$ & 5.916E2 & $6.667 \mathrm{E} 2$ & 1.185E3 & 300.8831 \\
\hline & HCQPSO & $6.880 \mathrm{E} 2$ & 1.195E3 & 1.637E3 & 236.6575 \\
\hline & SZOA & 5.799E2 & 5.799E2 & 5.799E2 & 0.0000 \\
\hline
\end{tabular}

Minimum, median, maximum, and standard deviation are respectively abbreviated as min, med, max, and std

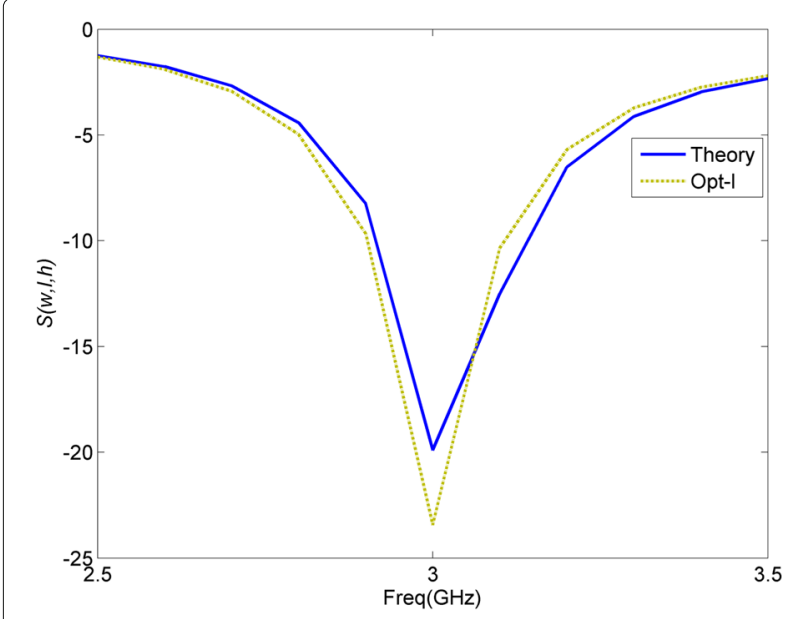

Fig. 4 Comparison of theoretical solution versus the solution through optimizing / by SZOA

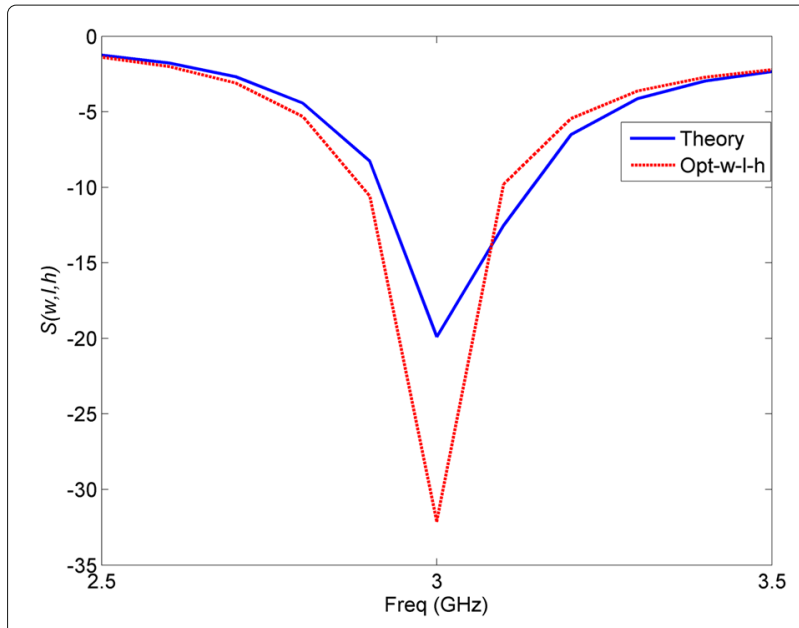

Fig. 5 Comparison of theoretical solution versus the solution through optimizing $w$, l, and $h$ by SZOA 


\section{Abbreviations}

CNrGA: Continuous non-revisiting genetic algorithm; HCQPSO: Hybrid chaotic quantum particle swarm optimization; PS: Pattern search; SZOA: Scan and zoom algorithm

\section{Acknowledgements}

Not applicable.

\section{Funding}

This research was supported in part by the National Natural Science Foundation of China (Project No. 61603275), the Applied Basic Research Program of Tianjin (15JCYBJC51500), and the Doctoral Fund Project of Tianjin Normal University (Project No. 043-135202XB1602, 52XB1505).

\section{Availability of data and materials}

Not applicable.

\section{Authors' contributions}

QZ implements the numerical simulation and collects the simulation results. XZ writes most of the paper. YL checks the numerical simulation and result analysis. All authors proofread the manuscript to reduce typos and grammar mistakes. All authors read and approved the final manuscript.

\section{Authors' information}

Qinglian Zhang majors in Communication Engineering in Tianjin Normal University (TJNU), Tianjin, China. She is working on antenna simulation and resonant frequency analysis.

Xin Zhang received his PhD in Electronic Engineering from City University of Hong Kong in 2013. Since 2015, he works as a lecturer at Tianjin Normal University. His main research interests are antenna design, resource allocation, evolutionary computation, and machine intelligence. He has published more than 40 technical papers on these subjects, including more than 30 papers in international journals.

Yupeng Li received the PhD degree in Communication and Information System in Beijing University of Posts and Telecommunications (BUPT), Beijing, China, in 2015. He is currently working as a lecturer in Tianjin Key laboratory of Wireless Mobile Communications and Power Transmission, Tianjin Normal University (TJNU), Tianjin, China. His current research interests include antenna design, advanced modulation scheme, and coherent optical communication system.

\section{Competing interests}

The authors declare that they have no competing interests.

\section{Publisher's Note}

Springer Nature remains neutral with regard to jurisdictional claims in published maps and institutional affiliations.

Received: 2 January 2018 Accepted: 11 March 2018

Published online: 20 March 2018

\section{References}

1. F Zhao, B Li, H Chen, X Lv, Joint beamforming and power allocation for cognitive MIMO systems under imperfect CSI based on game theory. Wirel. Pers. Commun. 73(3), 679-694 (2013)

2. Q Liang, X Cheng, S Samn, NEW: Network-enabled electronic warfare for target recognition. IEEE Trans. Aerosp. Electron. Syst. 46(2), 558-568 (2010)

3. Q Liang, X Cheng, S Huang, D Chen, Opportunistic sensing in wireless sensor networks: theory and applications. IEEE Trans. Comput. 63(8), 2002-2010 (2014)

4. HK Min, MS Song, I Song, JH Lim, A frequency-sharing weather radar network system using pulse compression and sidelobe suppression. EURASIP J. Wirel. Commun. Netw. 2016, 100 (2016)

5. Q Liang, Situation understanding based on heterogeneous sensor networks and human-inspired favor weak fuzzy logic system. IEEE Syst. J. 5(2), 156-163 (2011)

6. X Zhang, X Zhang, A novel artificial bee colony algorithm for radar polyphase code and antenna array designs. EURASIP J. Wirel. Commun. Netw. 2016(1), 40 (2016)

7. F Zhao, X Sun, H Chen, R Bie, Outage performance of relay-assisted primary and secondary transmissions in cognitive relay networks. EURASIP J. Wirel. Commun. Netw. 2014, 60 (2014)
8. F Zhao, W Wang, H Chen, Q Zhang, Interference alignment and game-theoretic power allocation in MIMO heterogeneous sensor networks communications. Signal Process. 126, 173-179 (2016)

9. X Zhang, SL Ho, WN Fu, Quantitative design and analysis of relay resonators in wireless power transfer system. IEEE Trans. Magn. 48(11), 4026-4029 (2012)

10. X Zhang, X Zhang, WN Fu, Fast numerical method for computing resonant characteristics of electromagnetic devices based on finite-element method. IEEE Trans. Magn. 53(6), 7401004 (2017)

11. F Zhao, L Wei, H Chen, Optimal time allocation for wireless information and power transfer in wireless powered communication systems. IEEE Trans. Veh. Technol. 65(3), 1830-1835 (2016)

12. J Zhu, B Hao, A new noninterior continuation method for solving a system of equalities and inequalities. J. Appl. Math. 2014, 592540 (2014)

13. ZZ Feng, L Fang, G He, An iteration primal-dual path-following method, based on wide neighbourhood and large update, for second-order cone programming. Optimization. 63(5), 679-691 (2014)

14. J Tang, G He, L Dong, L Fang, J Zhou, A smoothing newton method for the second-order cone complementarity problem. Appl. Math. 58(2), 223-247 (2013)

15. J Yu, M Li, Y Wang, G He, A decomposition method for large-scale box constrained optimization. Appl. Math. Comput. 231(12), 9-15 (2014)

16. X Jin, Y Liang, D Tian, F Zhuang, Particle swarm optimization using dimension selection methods. Appl. Math. Comput. 219(10), 5185-5197 (2013)

17. ZS Zhao, X Feng, YY Lin, F Wei, SK Wang, TL Xiao, MY Cao, ZG Hou, M Tan, Improved rao-blackwellized particle filter by particle swarm optimization. J. Appl. Math. 2013(302170) (2013)

18. S Pang, T Li, F Dai, M Yu, Particle swarm optimization algorithm for multisalesman problem with time and capacity constraints. Appl. Math. Inf. Sci. 7(6), 2439-2444 (2013)

19. Z Wu, X Xia, Optimal switching renewable energy system for demand side management. Sol. Energy. 114, 278-288 (2015)

20. B Wang, Z Wu, XXia, A multistate-based control system approach toward optimal maintenance planning. IEEE Trans. Control Syst. Technol. 25(1), 374-381 (2017)

21. F Zheng, C Han, Y Wang, Parallel SSLE algorithm for large scale constrained optimization. Appl. Math. Comput. 217(12), 5377-5384 (2011)

22. CY Han, FY Zheng, TD Guo, GP He, Parallel algorithms for large-scale linearly constrained minimization problem. Acta Math. Applicatae Sin. Engl. Ser. 30(3), 707-720 (2014)

23. B Sobhani, E Paolini, A Giorgetti, M Mazzotti, M Chiani, Target tracking for UWB multistatic radar sensor networks. IEEE J. Sel. Top. Signal Process. 8(1), 125-136 (2017)

24. X Gong, J Zhang, D Cochran, KXing, Optimal placement for barrier coverage in bistatic radar sensor networks. IEEE/ACM Trans. Networking. 24(1), 259-271 (2016)

25. Q Liang, X Cheng, KUPS: Knowledge-based ubiquitous and persistent sensor networks for threat assessment. IEEE Trans. Aerosp. Electron. Syst. 44(3), 1060-1069 (2008)

26. L Han, J Mu, Y Wang, J Gao, Performance analysis of multi-hop full-duplex decode-and-forward relaying. Ad Hoc Netw. 58, 54-61 (2017)

27. MJ Alam, MRI Faruque, MT Islam, Split quadrilateral multiband microstrip patch antenna design for modern communication system. IET Microwaves Antennas Propag. 11(9), 1317-1323 (2017)

28. X Chen, PY Qin, YJ Guo, G Fu, Low-profile and wide-beamwidth dual-polarized distributed microstrip antenna. IEEE Access. 5, 2272-2280 (2017)

29. NW Liu, L Zhu, X Zhang, WW Choi, A wideband differential-fed dual-polarized microstrip antenna under radiation of dual improved odd-order resonant modes. IEEE Access. 5, 23672-23680 (2017)

30. P Venkataraman, Applied Optimization with MATLAB Programming. (Wiley-Interscience, New york, 2009)

31. G Ma, B Huang, Optimization of process parameters of stamping forming of the automotive lower floor board. J. Appl. Math. 2014, 470320 (2014)

32. J Zhu, B Hao, A new class of smoothing functions and a smoothing newton method for complementarity problems. Optimization Letters. 7(3), 481-497 (2013)

33. J Tang, G He, L Fang, A new non-interior continuation method for second-order cone programming. J. Numer. Math. 21(4), 301-323 (2013) 
34. J Tang, G He, L Dong, L Fang, A new one-step smoothing newton method for second-order cone programming. Appl. Math. 57(4), 311-331 (2012)

35. L Sun, G He, Y Wang, C Zhou, An accurate active set newton algorithm for large scale bound constrained optimization. Appl. Math. 56(3), 297-314 (2011)

36. L Fang, C Han, A new one-step smoothing newton method for the second-order cone complementarity problem. Math. Meth. Appl. Sci. 34(3), 347-359 (2011)

37. J Tang, G He, L Dong, L Fang, A smoothing newton method for second-order cone optimization based on a new smoothing function. Appl. Math. Comput. 218(4), 1317-1329 (2011)

38. F Gunes, F Tokan, Pattern search optimization with applications on synthesis of linear antenna arrays. Expert Syst. Appl. 37(6), 4698-4705 (2010)

39. SY Yuen, CK Chow, A genetic algorithm that adaptively mutates and never revisits. IEEE Trans. Evol. Comput. 13(2), 454-472 (2009)

40. OE Turgut, Hybrid chaotic quantum behaved particle swarm optimization algorithm for thermal design of plate fin heat exchangers. Appl. Math. Model. 40(1), 50-69 (2016)

41. F Zhao, $\mathrm{H}$ Nie, $\mathrm{H}$ Chen, Group buying spectrum auction algorithm for fractional frequency reuses cognitive cellular systems. Ad Hoc Netw. $\mathbf{5 8}$ 239-246 (2017)

42. X Lu, H Wang, X Wang, On Kalman smoothing for wireless sensor networks systems with multiplicative noises. J. Appl. Math. 2012, 717504 (2012)

\section{Submit your manuscript to a SpringerOpen ${ }^{\circ}$ journal and benefit from:}

- Convenient online submission

Rigorous peer review

- Open access: articles freely available online

- High visibility within the field

- Retaining the copyright to your article

Submit your next manuscript at $>$ springeropen.com 